\title{
Effects of deformation dynamics in problems of heavy transport
}

\author{
Sergei V. Nosachev, Mikhail V. Chuveiko*, Maria Yu. Zhukova, Gennadiy A. Kuzin, and \\ Igor A. Khozyaev
}

Don State Technical University, 344000 Rostov-on-Don, Russia

\begin{abstract}
The problem of the appearance of the sliding effect in the wheel-road contact in railway transport is considered. It is known that the sliding effect accompanying the rolling process of the wheel is explained by two fundamentally different processes: sliding caused by the loss of traction of the wheel to the road surface and, so-called, deformation sliding. The phenomenon of deformation sliding is not related to loss of adhesion and can occur even when the wheel is fully engaged with the road surface. In fact, the deformation sliding is due to the non-trivial deformation dynamics of the rim of the wheel and the rail which results in an actual reduction or increase in the speed of the translational motion of the wheel while maintaining its angular frequency of rotation. Due to the fact that the phenomenon of loss of adhesion, as well as the accompanying sliding, has been studied in some detail, in this paper attention is focused specifically on the deformation component of the sliding process.
\end{abstract}

\section{Description of the mathematical model}

Numerous studies have been devoted to the problem of the dynamics of wheel-rail interaction [1-4]. In this case, the dynamic system is considered on the basis of an analysis of the dynamic system of the machine interacting with the dynamic coupling formed by tribo-conjugation in the contact region. In known studies, the dynamic characteristic of a friction unit is considered to be given, the parameters of which do not depend on the elastic properties of the wheel. In contrast to these studies, the proposed study proves that the properties of the frictional bond formed are not invariant to the elastic characteristics of the wheel. Thus, many known properties of the system are refined and supplemented.

Consider the problem of rolling the wheel along the road surface (see Figure 1). We will assume that the rigidity of the road is large enough, so that its deformations can be neglected. Here, as the wheel, an elastic rim fixed to the disk is considered. At the same time, the dynamics of radial deformations is not considered.

The wheel axis rotates at some fixed angular velocity $\omega$. External forces are applied to the wheel: the normal pressure $F_{n}$ (i.e., the force pressing the wheel to the road surface), the tangential force $F_{\tau}$ (the force preventing the movement of the wheel) and the force of contact interaction with the road surface. The forces of contact interaction with the surface

\footnotetext{
* Corresponding author: chuveyko@gmail.com
} 
of the road are determined by the processes occurring in the contact spot. According to modern ideas about the process of friction [10-12], we can distinguish two modes of tribocontact functioning: "adhesion mode " and " sliding mode ":

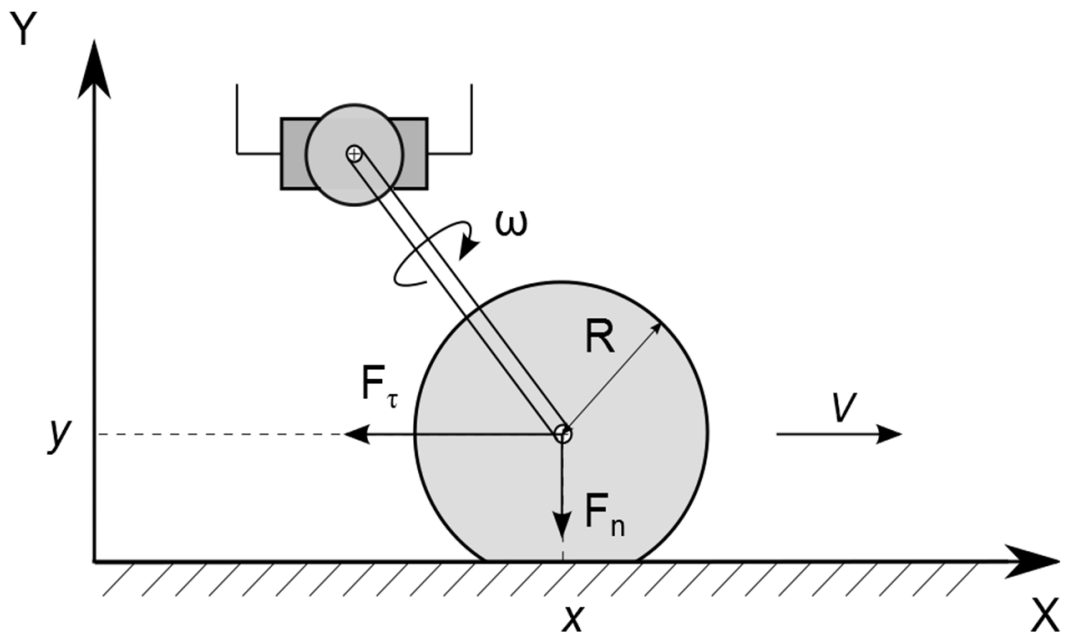

Fig. 1. Wheel-road surface system.

"Adhesion mode" is a mode in which stable bonds are established between the individual elements of the contacting surfaces in the contact zone. The presence of these constraints precludes mutual motion of the surfaces. In this mode of functioning, the tribological relationship can be described by means of the so-called force function that determines the force of the contact interaction, depending on the relative displacements. Studies show that the form of this function is identical to the strength function of Hooke.

"Sliding mode" is a mode in which the physical-mechanical connections described above do not have a permanent nature. In this case, the interacting surfaces perform a continuous motion relatively to each other. The emergence of contact forces is conditioned by the continuous process of formation and destruction of new bonds. The forces of contact interaction arising in this case are usually referred to as the frictional force of sliding. The magnitude of this force can be found by the formula.

The wheel itself is modeled by a set of sectors of the elastic rim fixed on an elastic disc. Thus, the mathematical model of the whole system is a set of partial differential equations. A more detailed derivation of model equations is given in [7-9].

$$
\left\{\begin{aligned}
\frac{d S}{d t} & =k_{2} \frac{\partial}{\partial l} \frac{1}{\tau}-h_{2} \frac{\partial}{\partial l}\left(\frac{1}{\tau^{2}} \frac{d \tau}{d t}\right)- \\
& -\frac{1}{H \bar{Q}}\left(k_{1} N+h_{1} \frac{d N}{d t}\right)+f(v) \\
\frac{d N}{d t} & =S-\bar{S} \\
\frac{d \tau}{d t} & =-\frac{1}{H \bar{Q}} \frac{\partial S}{\partial l} \\
\frac{d x}{d t} & =v \\
\frac{d v}{d t} & =\frac{1}{m}\left(\int_{D} f(v) d l-F_{n}\right)
\end{aligned}\right.
$$

where: $S$ - flow, $\mathrm{kg} / \mathrm{s} ; \bar{S}$ - Specified flow value, $\mathrm{kg} / \mathrm{s} ; H$ - area of section of the cylinder, $\mathrm{m}^{\wedge} 2 ; \bar{Q}$ - initial bulk density of the cylinder $\mathrm{kg} / \mathrm{m}^{\wedge} 3 ; k_{1}$ - specific tangential elasticity of the disc, $\mathrm{kg} /(\mathrm{s} 2 \cdot \mathrm{m}) ; h_{1}$ - specific tangential dissipation of the disk, $\mathrm{kg} /(\mathrm{s} \cdot \mathrm{m}) ; k_{2}-$ 
specific longitudinal elasticity, $(\mathrm{kg} \cdot \mathrm{m}) / \mathrm{s}^{\wedge} 2 ; h_{2}$ - specific longitudinal dissipation, $(\mathrm{kg} \cdot$ m) / s; $N$ - magnitude of tension, kg; $\tau$ - coefficient of compaction of the material; $x$ horizontal coordinate of the wheel center, $\mathrm{m} ; v$ - translational speed of the center of the wheel, $\mathrm{m} / \mathrm{s}$; - weight of the wheel, $\mathrm{kg} ; f(v)$ - specific force of contact interaction, $\mathrm{kg} / \mathrm{s}^{\wedge} 2$; $D$ - length of the contact spot, $\mathrm{m} ; F_{n}$ - force of external influence, $(\mathrm{kg} \cdot \mathrm{m}) / \mathrm{s}^{\wedge} 2$.

\section{Computer modelling}

From the classical assumptions of the wheel's dynamic, we should assume that the velocity of the center of the wheel should be determined by the product of the angular frequency by the radius. However, the simulation results showed otherwise. The actual speed of the center of the wheel is below the expected speed. This is due to deformation processes in the wheel. This phenomenon was called the "deformation sliding" [5-9]. In order to numerically characterize this phenomenon, we introduce a quantity - the coefficient of relative sliding:

$$
\gamma_{v}=\frac{v-\bar{v}}{v} \cdot 100 \%
$$

where: $v$ - translational speed of the center of the wheel, $\mathrm{m} / \mathrm{s}$;

$\bar{v}$ - the ideal translational velocity of the wheel center (determined by the product of the angular frequency of the wheel to its radius), $\mathrm{m} / \mathrm{s}$.

The numerical experiment gave the following dependence of the coefficient of relative sliding on the tangential load (see Fig.2).

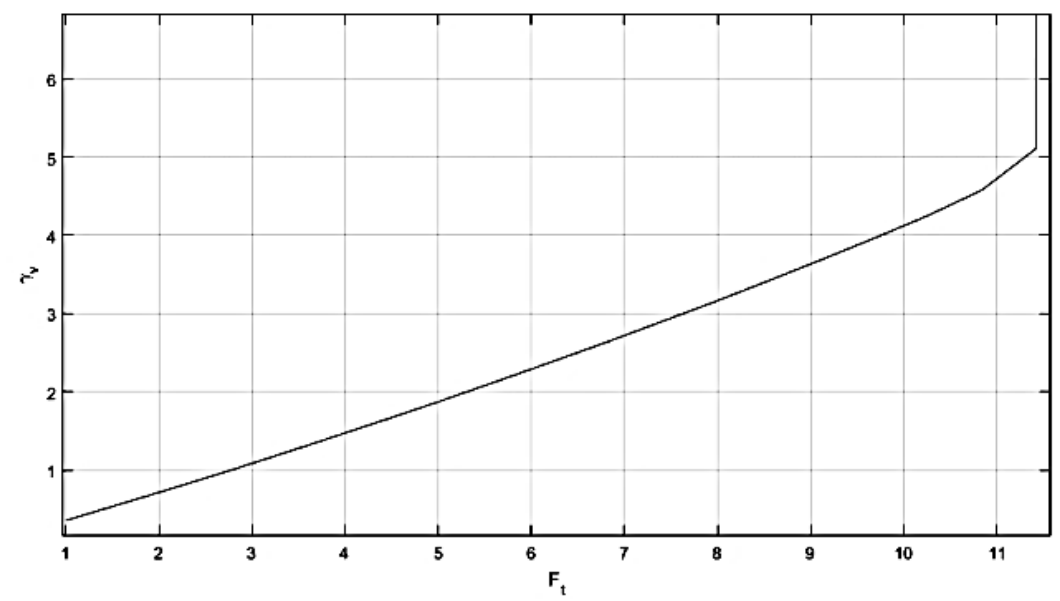

Fig. 2. Dependence of the coefficient of relative sliding on the load.

Here there is an almost linear dependence up to a certain threshold value. After which the coefficient rises sharply, which is explained by the total loss of adhesion. The slope of the linear part of the graph is mainly determined by the stiffness characteristics of the wheel.

Also, interesting to consider the graph demonstrates the distribution of the tangential speed of the wheel rim elements over its surface (see Fig. 3). A similar nature of the velocity distribution is typical for the rolling of a deformable wheel in the mode of full engagement with the road surface. In addition, the graph shows two values: the actual speed of the centre of the wheel and the specified speed, that is, the speed determined by the classical formula: $R \omega$. 


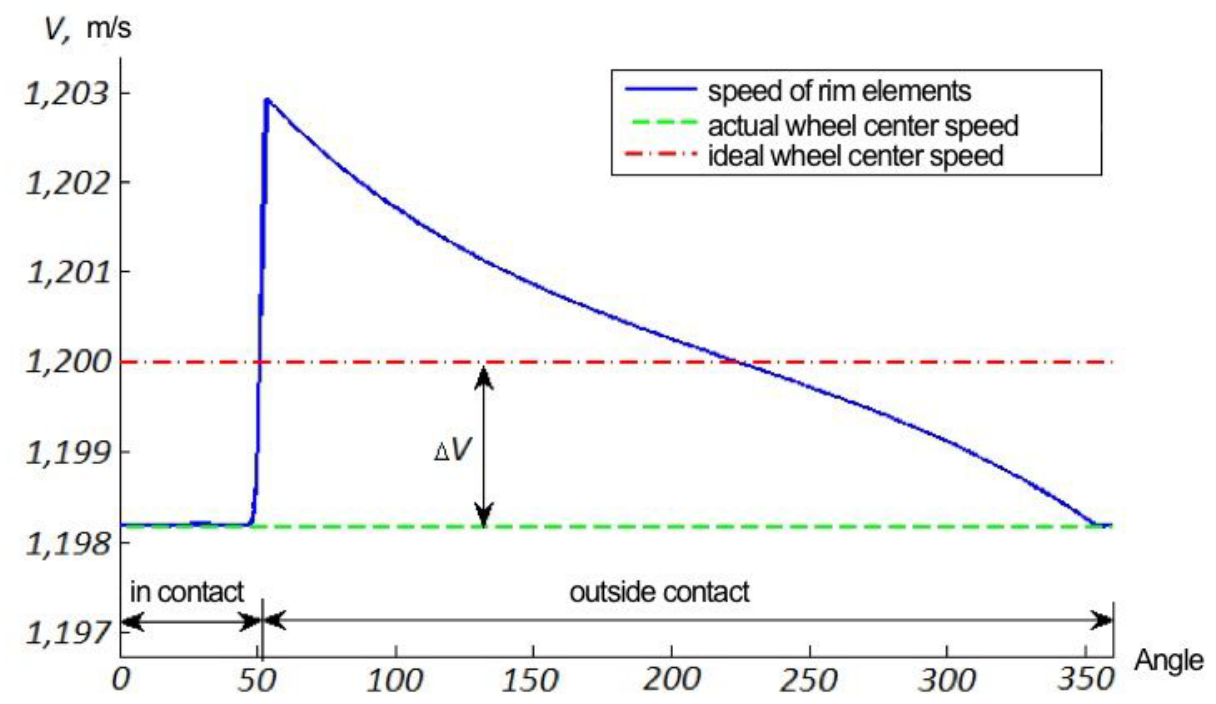

Fig. 3. The distribution of the tangential speed of the wheel rim elements over its surface.

It is easy to see that the $\bar{v}>v$ relation holds, which leads to the appearance of some nonzero "slip" value, characterized by the difference in velocities:

$$
\Delta V=v-\bar{v}
$$

However, unlike classical sliding, the surfaces of the wheel and the road in the zone of their interaction remain stationary relative to each other, i.e. there are stable bonds. This fact is clearly demonstrated by the equality $v=\bar{v}$ that takes place in the contact zone. The reason for the appearance of some nonzero quantity $\Delta V$ is the deformation processes occurring in the wheel. According to the results of computer simulation, the dynamics of deformation processes has a rather complex character of behaviour, caused by a large number of external factors and characteristics of the wheel-road dynamic system. However, despite this fact, the dependence on the magnitude of the external load has a character close to linear.

\section{The conclusion}

The original mathematical model of the "wheel-rail" system is presented. This mathematical model is described by a system of partial differential equations. It allows us to consider the elastic-dissipative properties of the wheel, as well as the peculiarities of the interaction of the contacting surfaces in the zone of the contact spot.

The mathematical model of the system made it possible to investigate the deformation processes occurring in the wheel during its rolling and to detect the phenomena caused by this process, such as: "elastic sliding", the emergence of a stable oscillatory process in certain operating modes.

Studies have shown that the actual speed of the wheel depends on the tangential load applied to it. This fact can be of particular importance in calculating the dynamics of motion of traction wheels of heavy transport. The forces of inertia at the stage of acceleration / deceleration of such systems acquire rather high values and can have a significant effect on the actual speed of motion. 


\section{References}

1. R.V. Chernov, Modern anti-skid protection on electric locomotives (The Urals Electromech. Institute of Engineering, Transp., 1977)

2. V.V. Deev, G.A. Ilyin, GS Afonin, Draft of trains (Transp., Moscow, 1987)

3. K. Johnson, Mechanics of contact interaction (Mir, Moscow, 1989)

4. V.K. Garg, R.V. Dukkipati, Dynamics of rolling stock (Transp., Moscow, 1988)

5. F.T. Barweli, R.G. Woolacott, Proc. Instit. of Mechanical Engineers, 177, 145 (1963)

6. A.Ya. Kogan, Vniizht bulletin, 5, 33 (2004)

7. M.V.Chuveyko, S.V.Nosachev, Bulletin of the Don state tech. univ., 78, 127 (2014)

8. M.V. Chuveyko, S.V. Nosachev, Modern trends of development of science and tech., 2-3, 112 (2017)

9. M.V. Chuveyko, S.V. Nosachev, MATEC Web of Conferences., 132, 02004 (2016)

10. A.Yu. Ishlinsky, I.V. Kragelsky, Technical Physics, 45, 276 (1944)

11. L. Bureau, Comptes Rendus de l'Académie des Sciences, 2, 695 (2001)

12. V.L. Veits, M.S. Bundur, V.E. Hitrik, J Frict Wear, 6, 653 (1985) 